\title{
Optimal cut-off criteria for duplex ultrasound for the diagnosis of restenosis in stented carotid arteries: Review and protocol for $a$ diagnostic study Paul J Nederkoorn*1 and Martin M Brown²
}

Address: ${ }^{1}$ Department of Neurology, Academic Medical Center, Amsterdam, The Netherlands and ${ }^{2}$ UCL Institute of Neurology, the National Hospital for Neurology and Neurosurgery, London, UK

Email: Paul J Nederkoorn* - p.j.nederkoorn@amc.uva.nl; Martin M Brown - m.brown@ion.ucl.ac.uk

* Corresponding author

Published: 22 July 2009

BMC Neurology 2009, 9:36
Received: 4 May 2009

Accepted: 22 July 2009

This article is available from: http://www.biomedcentral.com/147/-2377/9/36

(c) 2009 Nederkoorn and Brown; licensee BioMed Central Ltd.

This is an Open Access article distributed under the terms of the Creative Commons Attribution License (http://creativecommons.org/licenses/by/2.0), which permits unrestricted use, distribution, and reproduction in any medium, provided the original work is properly cited.

\begin{abstract}
Background: Carotid angioplasty with stenting is a relatively new, increasingly used, less-invasive treatment for the treatment of symptomatic carotid artery stenosis. It is being evaluated in ongoing and nearly finished randomized trials. An important factor in the evaluation of stents is the occurrence of in-stent restenosis. An un-stented carotid artery is likely to have a more elastic vessel wall than a stented one, even if stenosis is present. Therefore, duplex ultrasound cut-off criteria for the degrees of an in-stent stenosis, based on blood velocity parameters, are probably different from the established cut-offs used for un-stented arteries. Routine criteria can not be applied to stented arteries but new criteria need to be established for this particular purpose.

Methods/Design: Current literature was systematically reviewed. From the selected studies, the following data were extracted: publication year, population size, whether the study was prospective, which reference test was used, and if there was an indication for selection bias and for verification bias in particular. Previous studies often were retrospective, or the reference test (DSA or CTA) was carried out only when a patient was suspected of having restenosis at DUS, which may result in verification bias.

Results: In general, the cut-off values are higher than those reported for unstented arteries. Previous studies often were retrospective, or the reference test (DSA or CTA) was carried out only when a patient was suspected of having restenosis at DUS, which may result in verification bias.

Discussion: To address the deficiencies of the existing studies, we propose a prospective cohort study nested within the International Carotid Stenting Study (ICSS), an international multi-centre trial in which over I,700 patients have been randomised between stenting and CEA. In this cohort we will enrol a minimum of 300 patients treated with a stent. All patients undergo regular DUS examination at the yearly follow-up visit according to the ICSS protocol. To avoid verification bias, an additional computed tomography angiography (CTA) will be performed as a reference test in all consecutive patients, regardless of the degree of stenosis on the initial DUS test.
\end{abstract}




\section{Background}

Carotid endarterectomy (CEA) is an effective and established treatment for secondary prevention of stroke in patients with symptomatic carotid artery stenosis[1]. Carotid angioplasty with stenting (CAS) is a relatively new, increasingly used, less-invasive treatment, which is being evaluated in ongoing or nearly finished randomized trials, such as the Carotid Revascularization Endarterectomy versus Stent Trial (CREST) and the International Carotid Stenting Study (ICSS) [2,3]. ICSS finished randomization and will publish its safety results in the short term. Previous trials that compared CEA with CAS were rather heterogeneous and not large enough to allow reliable conclusions. Furthermore, because there is limited follow-up information to date, the long-term effect of CAS remains unclear. We therefore need more data from these nearly finished randomised trials, including long followup, before recommending if and when stenting should replace endarterectomy in clinical practice $[4,5]$.

An important factor in the evaluation of stents is the occurrence of in-stent restenosis. Both the Carotid and Vertebral Artery Transluminal Angioplasty Study (CAVATAS) and the Stent-Protected Angioplasty versus Carotid Endarterectomy (SPACE) study reported a higher incidence of restenosis in patients treated with CAS than in patients treated with CEA $[6,7]$. However, we do not know if an in-stent restenosis will give rise to symptoms in a similar way to atherosclerotic carotid stenosis, and the clinical consequences of restenosis are yet unknown. In CAVATAS the majority of patients in the endovascular arm were treated by angioplasty without stenting. The SPACE-investigators concluded that it could not be excluded that the degree of in-stent stenosis was slightly overestimated by conventional ultrasound criteria. Long term follow-up data of patients treated with a stent are needed to investigate the clinical consequences of in-stent restenosis. Prior to these analyses, to reliably diagnose instent restenosis, we first need reliable duplex ultrasound cut-off criteria.

Traditionally, the degree of stenosis in an untreated carotid artery was measured with conventional digital subtraction angiography (DSA). Because of a small but non-negligible risk of stroke or death, DSA has been replaced by non-invasive tests, such as duplex ultrasound (DUS), CT angiography (CTA) or MR angiography (MRA) [8-10]. In the follow-up of patients with a stent, DUS is often used to monitor the patency of the stent and the occurrence of in-stent restenosis. For routine evaluation of un-stented carotid arteries, DUS is a well validated diagnostic test and the cut-off criteria for the different degrees of stenosis are clear $[8,9]$. For measurements within stents, however, these criteria may not suffice.
In a stenosed artery, narrowing of the lumen results in higher blood flow velocities at that point. Estimating the degree of stenosis with DUS is based on this principle. The peak systolic velocity (PSV) is the best predictor for the severity of the stenosis [11]. However, the degree of restenosis in a stented carotid artery, measured according to the North American Symptomatic Carotid Endarterectomy Trial (NASCET) criteria on CTA or MRA, is often less severe than expected based on the PSV in the DUS test. Possibly, blood flow and blood turbulence behave differently in an artificial stent than in a normal vessel. This problem has been addressed in literature before; particularly by Lal en coworkers [12]. An un-stented carotid artery is likely to have a more elastic vessel wall than a stented one, even if stenosis is present. The cut-off criteria for the degrees of an in-stent stenosis, based on blood velocity parameters, are probably different from the established cut-offs used for un-stented arteries. We hypothesise that the PSV raises more in a stented than in a unstented carotid artery with a similar degree of stenosis (figure 1). Additional duplex characteristics may also be different in an in-stent restenosis, such as formation of
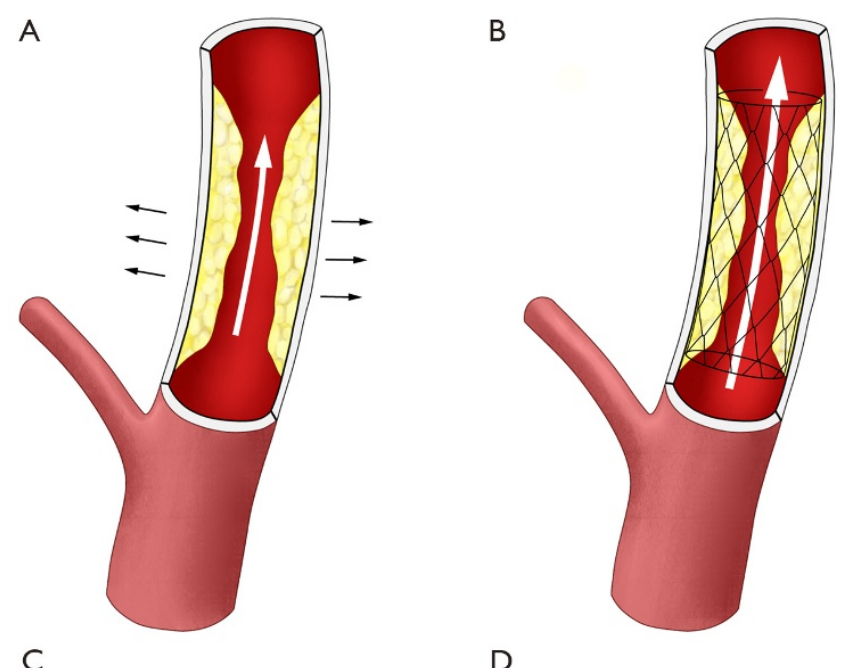

C
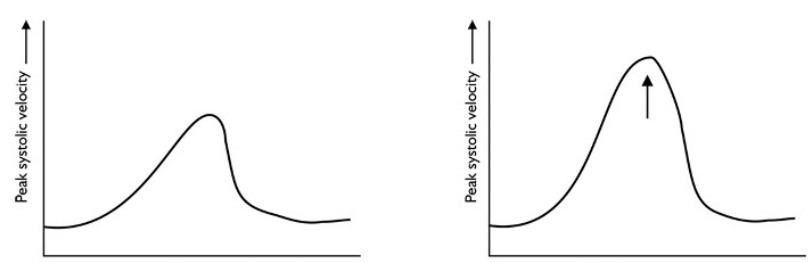

\section{Figure I}

Illustration of our hypothesis that a un-stented carotid artery (A) has a more elastic vessel wall than a stented one (B), and that the PSV raises more in a stented (D) than in an un-stented carotid artery (C) with a similar degree of stenosis. 
intimal hyperplasia instead of atherosclerotic plaque as cause of the stenosis. These characteristics need to be studied as well; the present study, however, will be limited to definition of PSV criteria. Routine criteria can not be applied to stented arteries but new criteria need to be established for this particular purpose.

Valid criteria are needed for future research and clinical decisions in patients treated with a carotid artery stent. In this article, we therefore first review the current literature and discuss the most important limitation of earlier diagnostic studies on this topic, verification bias. In the discussion, we describe the design of a new diagnostic study designed to validate the use of DUS in-stent stenosis measurements during follow-up after CAS and to determine reliable cut-off criteria for the different degrees of stenosis.

\section{Methods/Design}

The PubMed databases have been searched from 2000 until 2009 for publications with "duplex ultrasound" combined with "carotid", "stent" or "in-stent", and "restenosis" as keywords, without language restrictions. Crossreferences and review articles were used for search completion. In case of more than one publication on this topic by one group, the most recent or largest series was chosen. A hand-search of relevant journals and conference proceedings was not performed. Based on titles and abstracts, studies evaluating duplex ultrasound for assessment of insent restenosis were selected. To be included in this review, the study needed to provide duplex ultrasound cut-off criteria, calculated by comparison with stenosis measurements on a reference test (CTA or DSA). From the selected studies, the following data were extracted: publication year, population size, whether the study was prospective, which reference test was used, and if there was an indication for selection bias and for verification bias in particular. A formal and systematic review, and meta-analysis, will be performed after the presented new diagnostic study is finished; including its results.

\section{Results}

We identified 6 unique diagnostic series on in-stent stenosis measurements with DUS compared to a reference test (CTA or DSA) [13-18]. In one study DUS and CTA were compared for in-stent measurements, but this paper needed to be excluded because no new criteria were calculated [18]. Additional file 1: Table S1 summarises the 5 series that propose new criteria. In general, the cut-off values are higher than those reported for unstented arteries. For example, the PSV cut-off value for the diagnosis of a $>70 \%$ stenosis varies between 300 and $450 \mathrm{~cm} / \mathrm{sec}$. The reported prevalence of in-stent restenosis was low, which is likely to reflect limited follow-up. Furthermore, the reference test was only performed in these previous studies if stenosis was found on DUS, introducing verification bias. Lal et al recently reported a relatively large population of 255 CAS procedures [14]. They confirm overestimation of the degree of in-stent restenosis if regular DUS criteria are applied. Comparisons were made with CTA and DSA. During follow-up, patients underwent DSA only if they were suspected as having restenosis on DUS. Criteria calculated from these data may suffer from verification bias. All patients in their series underwent CTA at the end of their follow-up. The latter data should not suffer from verification bias. In another recent series of Aburahma et al, selection was made with DUS and only patients with symptomatic $\geq 50 \%$ stenosis or asymptomatic $\geq 80 \%$ stenosis were included in their diagnostic study [13]. Applying their DUS criteria to all stented patients is probably not correct because of the selection criteria applied. Zhou et al published a large series but concluded that they infrequently found cases of severe stenosis after CAS, and that a multicentre study is warranted to establish reliable instent DUS criteria [15]. Only Kwon et al reported a series of patients all undergoing both DUS and the reference test, CTA. This study, however, was too small $(n=27)$ to provide new in-stent cut-off criteria [18].

It is clear from the published data that only small and preselected populations with in-stent restenosis have been studied to date. A large study with sufficient patients with restenosis is therefore needed, as authors of the listed papers also recognised.

\section{Verification bias}

Previous studies often were retrospective, or the reference test (DSA or CTA) was carried out only when a patient was suspected of having restenosis at DUS, which may result in verification bias. Verification bias is introduced if the decision to perform the reference standard procedure depends on the results of the test under investigation, precluding a reliable estimate of the diagnostic accuracy of the latter [19]. The effect of verification bias is explained in figure 2. In a diagnostic study in carotid artery stenosis, in order to obtain valid results, during the follow-up all patients need to undergo both the reference test and the test under investigation, regardless of the degree of stenosis on the initial DUS test. If patients were selected based on duplex cut-offs before treatment with CAS, this does not influence the results. On the contrary, the population selected based on age, gender, symptoms, and degree of stenosis prior to treatment, is exactly the domain for a diagnostic study with this purpose.

\section{Protocol for proposed diagnostic study}

To address the deficiencies of the existing studies, we plan to conduct a prospective cohort study nested within the International Carotid Stenting Study (ICSS), an international multi-centre trial in which over 1,700 patients with 


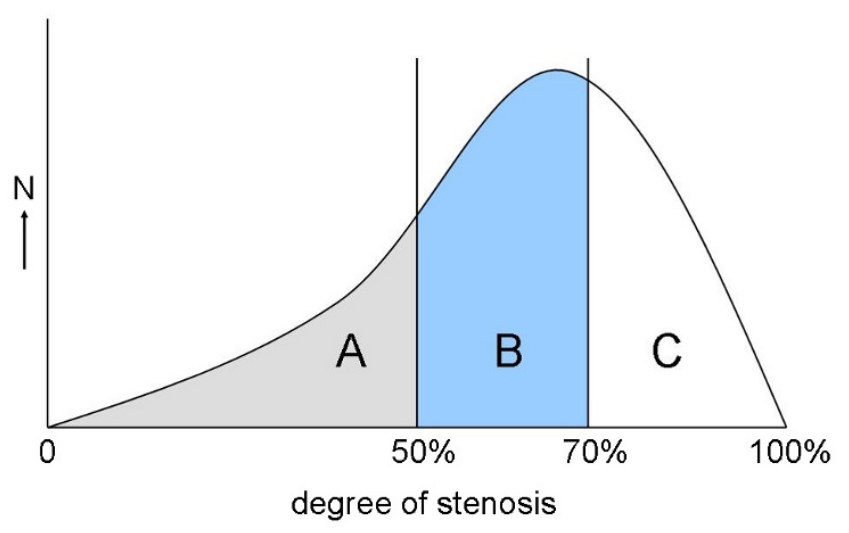

Figure 2

Example of the effect of verification bias in a hypothetical true distribution of degree restenosis in patients treated with a carotid artery stent. If a DUS cut-off value of for example $50 \%$ stenosis is used to select patients for a diagnostic study, only the patients in part B and $C$ are included and will undergo the reference test. Patients in part $A$ are excluded. The number of patients below a certain threshold, for example $70 \%$ is too low (proportion $B$ instead of proportion $A$ and $B$ ) and therefore leads to incorrect estimates of the diagnostic value, the specificity in particular. Therefore, DUS criteria obtained from a diagnostic study with comparison with the reference test in part B and $C$, can not be applied to routine clinical practice for all stented patients (part A, B, and C).

recently symptomatic internal carotid stenosis have been randomised in equal proportions between stenting and endarterectomy. The ICSS completed recruitment at the end of 2008. The trial protocol specifies annual clinical and ultrasound follow up. A sufficiently large population can be enrolled and because the study is embedded in the ICSS, long term follow-up is guaranteed, allowing us to obtain a larger number of restenoses and therefore more precise estimates for DUS cut-off criteria. Most importantly, during the follow-up all patients in the sub-study will undergo both the reference test of CTA and the test under investigation, regardless of the degree of stenosis on the initial DUS test.

\section{Reference test}

In the protocol, we will use CTA as the reference test for in-stent stenosis measurements. A diagnostic test that provides clear images of the lumen of the internal carotid artery is crucial, because a NASCET-like stenosis measurement is necessary as a reference to estimate the optimal PSV cut-offs for DUS. DSA would be preferable for optimal images of the lumen of stented (carotid) arteries. However, in our opinion, this test would not be ethical anymore as reference test in this diagnostic study because of the small but not negligible complication rate. CTA and MRA are non- or minimally invasive tests providing good lumen images, enabling a 'NASCET'-like stenosis measurement. MRA is not suitable for visualisation of the lumen in a stent, due to artefacts in the magnetic field caused by the material of the stent. Therefore, CTA is the better choice. The use of intravenous iodinated contrast in CTA allows excellent images of the lumen of the arteries. To date, CTA offers high spatial resolution and contrast resolution, and it is a fast technique. Currently, the quality of imaging of the lumen of the artery with CTA is comparable to DSA. We realise that CTA is better validated for non-stented than for stented (carotid) arteries. The diagnostic accuracy of CTA compared to DSA, to diagnose a $70-99 \%$ stenosis, was calculated in several studies $[8,10]$. Wardlaw et al., in a recent meta-analysis, reported a sensitivity and specificity of 77\% (95\% CI: $68-84 \%$ ) and 95\% (95\%CI: 91-97\%) respectively [8]. Also, CTA may have certain pitfalls in measuring in-stent stenosis like blurring artefacts in the stent. However, given the limitations, we think CTA is the best choice as a reference test in the assessment of stenosis measurements [20].

Crucial is the fact that we can compare DUS results to a technique that provides clear images of the lumen, in order to investigate if the PSV raises more in a stented artery than in an unstented artery when the remaining lumen is the same as. Because patients will be recruited from multiple centres, the CT scanners will not be identical and we can only handle this limitation by using comparable scan protocols and similar post-processing techniques. Also, different types of stents will be used. If these stents do not have the same physical properties, the PSV's are perhaps slightly different as well [21]. We shall collect data about the used stents, and in our analyses we shall investigate if there is a relation between type of stent and blood flow parameters. However, the advantage of a multi-centre study is that the results will be widely applicable.

\section{Patients}

The diagnostic tests will be performed during routine follow-up of the ICSS trial [3]. The inclusion and exclusion criteria are listed in Table 1. In short, all patients with a symptomatic atheromatous carotid stenosis, $\geq 50 \%$ by NASCET criteria, suitable for stenting and surgical endarterectomy, can be included for treatment. In addition to the general ICSS criteria, patients are excluded if they have a contraindication for the contrast agent used for the CTA, such as renal failure. In the ICSS, patients have scheduled follow-up visits at 30 days after treatment, 6 months after randomisation and then annually. We will ask all patients who received a stent to participate in this diagnostic study at their follow-up visit 1 year after treatment, or, for 
Table I: Inclusion criteria International Carotid Stenting Study (ICSS)

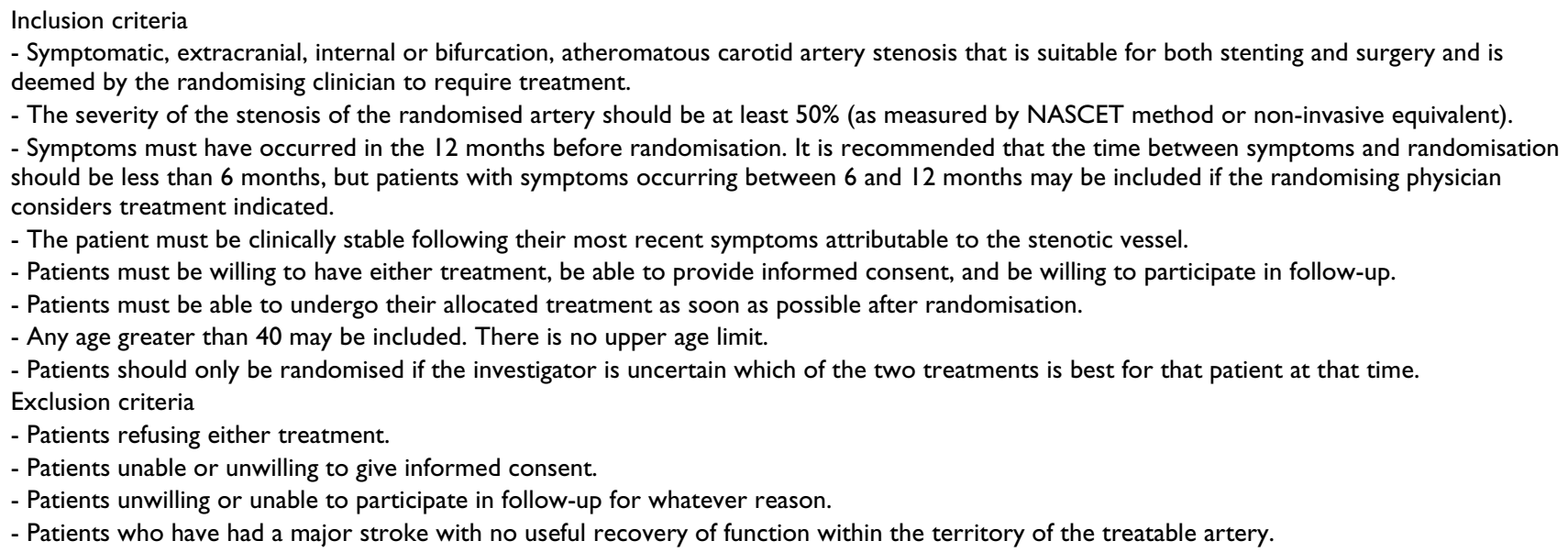

patients included more than one year ago, at the first (yearly) ICSS follow-up visit thereafter. A separate informed consent for this diagnostic sub-study informing the patient about the risks of the extra CTA test is obtained. The sub-study has received approval from the Multicentre Research Ethics Committee in the UK. The complete protocol of ICSS and of the present diagnostic sub study is available at: http://www.ion.ucl.ac.uk/ cavatas icss/icss.htm.

\section{Sample size}

In a sample size calculation in a diagnostic study, in addition to an estimation of the prevalence of disease, a precision of the diagnostic accuracy needs to be defined. We estimate a prevalence of 10 to $20 \%$ in-stent restenosis $(\geq 50 \%)$ one year after treatment with a stent. To obtain estimates of sensitivity of approximately $90 \%$ with a confidence interval of maximally $10 \%$, and a prevalence of restenosis after one year of $20 \%$, we would require a minimum of 172 patients [22]. The estimate of $20 \%$ re-stenosis is at the upper limit of the values reported in literature. We used this estimate, because our follow-up interval (after 1 year or more) will be later than of most published numbers. If we use $10 \%$ restenosis in the sample size calculation, with similar estimates for sensitivity, specificity, and the confidence interval(s), the number exceeds 300 patients (approximately 350). Because the ICSS included more than 800 patients treated with a stent, and because the follow-up of the ICSS will continue for several years, we expect inclusion of a sufficient high number of patients.

\section{Diagnostic tests}

During the DUS examination, different blood flow velocity parameters will be recorded. The peak systolic velocity (PSV) in the stent will be used as our outcome variable because it is considered the most accurate estimator of the degree of stenosis for DUS [11]. CTA is used as the reference test for in-stent stenosis measurements. Because different centres participate, we will work with different CT machines. However, we will match the protocols. Most importantly, for the assessment of the degree of stenosis, multiplanar (MPR) or curved planar reconstructions (CPR) need to be made [20].

\section{Stenosis measurement}

Two independent observers, blinded for clinical information and for the results of the other diagnostic tests, will perform a stenosis measurement on the reconstructed CTA images, following the Standards for Reporting of Diagnostic Accuracy (STARD) guidelines [23]. The grade of stenosis will be measured according to the NASCET criteria [1]. Stenosis measurements in NASCET were done with DSA. The degree of stenosis was defined as the diameter of the remaining lumen at the stenosis as percentage of the normal lumen distal to the stenosis. The projection, lateral, posteroanterior, or oblique, which shows the most severe stenosis, is used for establishing the degree of stenosis. Measurements with CTA should preferable be done in a comparable manner, in order to correctly apply the trial-data to the clinical decisions about CEA. Thus, for a valid comparison with DSA, the percentage of stenosis will be measured on the MPR or CPR post-processed images of the internal carotid artery, using the three projections mentioned above only.

\section{Data analysis}

Receiver operating characteristics (ROC) curves will be constructed for the diagnoses of $70 \%-99 \%$ and $50 \%-$ $69 \%$ stenosis. The associated optimal sensitivities, specificities, and peak-systolic-velocity thresholds will be derived from the ROC curves. The main result of the analyses will be optimal cut-off points of duplex for in-stent 
restenosis (not the diagnostic accuracy of duplex) within the context of the present study.

\section{Meta-analysis}

When the present study is completed, we will pool our data with the other diagnostic studies on this particular topic available in literature [13-18]. In addition to pooled weighted estimates of the overall diagnostic accuracy, the data will be modelled using summary ROC analyses. With this model it will be possible to investigate the effect of important quality criteria of the individual studies on the diagnostic accuracy, such as sample size, duration of follow-up, and presence of verification bias.

\section{Discussion}

Carotid angioplasty with stenting (CAS) is being evaluated in ongoing randomized trials. An important factor in the evaluation of carotid artery stents is the degree of possible in-stent restenosis. DUS is a fast and easy test to asses the degree of stenosis. Whereas this diagnostic tool is well validated for stenosis measurements in un-stented carotid arteries, precise cut-off criteria for stented carotid arteries are not available yet. The aim of the proposed study is to validate the use of DUS for in-stent stenosis measurements during follow-up after CAS and to determine reliable cut-off criteria for the different degrees of stenosis. Valid criteria are needed for future research and clinical decision making in patients treated with a carotid artery stent. In conclusion, the ongoing ICSS study provides a unique opportunity to obtain valid DUS in-stent criteria in a sufficiently large population, with a long follow-up and expected high prevalence of restenosis. Verification bias will be avoided by performing both DUS and CTA in all patients. Afterwards, our data will be pooled with other studies, taking into account the limitations of individual studies.

\section{Abbreviations}

CAS: carotid angioplasty with stenting; CEA: carotid endarterectomy; CTA: CT angiography; DUS: duplex ultrasound; PSV: peak systolic velocity; ICSS: International Carotid Stenting Study.

\section{Competing interests}

The authors declare that they have no competing interests.

\section{Authors' contributions}

PJN has designed the sub study and wrote the manuscript. MMB has corrected and approved the study protocols and all versions of the current paper. Both authors read and approved the final manuscript.

\section{Authors' information}

PJN is Neurologist and Clinical Epidemiologist and did previous research in the field of carotid artery stensosis; with special interest in diagnostic study design. $M M B$ is Professor of Stroke Medicine and Consultant Neurologist and has a large track record in stroke research and previous randomised trials; currently he is principal investigator of the International Carotid Stenting Study.

\section{Additional material}

\section{Additional file 1}

Table S1. previously reported DUS cut-off values for stenosis measurements within a stent.

Click here for file

[http://www.biomedcentral.com/content/supplementary/14712377-9-36-S1.doc]

\section{Acknowledgements}

ICSS has been funded by grants from the Medical Research Council, The Stroke Association, Sanofi Synthelabo and the European Commission. MMB's Chair in Stroke Medicine at University College London is supported by the Reta Lila Weston Trust for Medical Research. Part of this work was undertaken at UCLH/UCL who received a proportion of funding from the Department of Health's NIHR Biomedical Research Centres funding scheme.

\section{References}

I. Rothwell PM, Eliasziw M, Gutnikov SA, Fox AJ, Taylor DW, Mayberg MR, Warlow CP, Barnett HJ, Carotid Endarterectomy Trialists' Collaboration. Carotid Endarterectomy Trialists' Collaboration: Analysis of pooled data from the randomised controlled trials of endarterectomy for symptomatic carotid stenosis. Lancet 2003, 361:107-16.

2. Hobson RW II: Update on the Carotid Revascularization Endarterectomy vs. Stent Trial (CREST) protocol. J Am Coll Surg 2002, 194( ( SuppI):S9-SI4.

3. Featherstone RL, Brown MM, Coward LJ, ICSS Investigators: International carotid stenting study: protocol for a randomised clinical trial comparing carotid stenting with endarterectomy in symptomatic carotid artery stenosis. Cerebrovasc Dis 2004, 18:69-74.

4. Brown MM, Hacke W: Carotid artery stenting: the need for randomised trials. Cerebrovasc Dis 2004, 18:57-6I.

5. Brown MM: Should carotid stenting replace carotid endarterectomy in routine clinical practice? Cerebrovasc Dis 2004, 18:69-74.

6. McCabe DJ, Pereira AC, Clifton A, Bland JM, Brown MM, CAVATAS Investigators: Restenosis after carotid angioplasty, stenting, or endarterectomy in the Carotid and Vertebral Artery Transluminal Angioplasty Study (CAVATAS). Stroke 2005, 36:28I-6.

7. Eckstein HH, Ringleb P, Allenberg JR, Berger J, Fraedrich G, Hacke W, Hennerici M, Stingele R, Fiehler J, Zeumer H, Jansen O: Results of the Stent-Protected Angioplasty versus Carotid Endarterectomy (SPACE) study to treat symptomatic stenoses at 2 years: a multinational, prospective, randomised trial. Lancet Neurol 2008, 7:893-902.

8. Wardlaw JM, Chappell FM, Best JJ, Wartolowska K, Berry E, NHS Research and Development Health Technology Assessment Carotid Stenosis Imaging Group: Non-invasive imaging compared with intra-arterial angiography in the diagnosis of symptomatic carotid stenosis: a meta-analysis. Lancet 2006, 367:1503-12.

9. Nederkoorn PJ, Graaf Y van der, Hunink MG: Duplex ultrasound and magnetic resonance angiography compared with digital subtraction angiography in carotid artery stenosis: a systematic review. Stroke 2003, 34:|324-31.

10. Koelemay MJ, Nederkoorn PJ, Reitsma JB, Majoie CB: Systematic review of computed tomographic angiography for assessment of carotid artery disease. Stroke 2004, 35:2306-12. 
II. Hunink MG, Polak JF, Barlan MM, O'Leary DH: Detection and quantification of carotid artery stenosis: efficacy of various doppler velocity parameters. AJR Am J Roentgenol 1993, 160:619-625.

12. Lal BK, Hobson RW 2nd, Goldstein J, Chakhtoura EY, Durán WN: Carotid artery stenting: is there a need to revise ultrasound velocity criteria? J Vasc Surg 2004, 39:58-66.

13. Aburahma AF, Abu-Halimah S, Bensenhaver J, Dean LS, Keiffer T, Emmett M, Flaherty S: Optimal carotid duplex velocity criteria for defining the severity of carotid in-stent restenosis. J Vasc Surg 2008, 48:589-94.

14. Lal BK, Hobson RW 2nd, Tofighi B, Kapadia I, Cuadra S, Jamil Z: Duplex ultrasound velocity criteria for the stented carotid artery. J Vasc Surg 2008, 47:63-73.

15. Zhou W, Felkai DD, Evans M, McCoy SA, Lin PH, Kougias P, El-Sayed $H F$, Lumsden $A B$ : Ultrasound criteria for severe in-stent restenosis following carotid artery stenting. J Vasc Surg 2008, 47:74-80.

16. Chi YW, White CJ, Woods TC, Goldman CK: Ultrasound velocity criteria for carotid in-stent restenosis. Catheter Cardiovasc Interv 2007, 69:349-354.

17. Stanziale SF, Wholey MH, Boules TN, Selzer F, Makaroun MS: Determining in-stent stenosis of carotid arteries by duplex ultrasound criteria. J Endovasc Ther 2005, 12:346-353.

18. Kwon BJ, Jung C, Sheen $\mathrm{SH}$, Cho JH, Han MH: CT angiography of stented carotid arteries: comparison with Doppler ultrasonography. J Endovasc Ther 2007, 14:489-97.

19. Begg CB, Greenes RA: Assessment of diagnostic tests when disease verification is subject to selection bias. Biometrics 1983, 39:207-2I5.

20. Nederkoorn PJ, Majoie CB, Stam J: Computed tomographic angiography of carotid artery stenosis. Carotid diseases, the role of imaging in diagnosis and management. Volume Chapter 12. Edited by: Gillard J. Cambridge University Press; 2007:I58-165.

21. Spies C, Doshi R, Spoon J, Snell RJ: Carotid artery stent type influences duplex ultrasonography derived peak systolic velocity: findings of an in-vitro model. Catheter Cardiovasc Interv 2007, 70:309-15.

22. Flahault A, Cadilhac M, Thomas G: Sample size calculation should be performed for design accuracy in diagnostic test studies. J Clin Epidemiol 2005, 58:859-62.

23. Bossuyt PM, et al.: Towards complete and accurate reporting of studies of diagnostic accuracy: The STARD. Ann Intern Med 2003, 138:40-4.

\section{Pre-publication history}

The pre-publication history for this paper can be accessed here:

http://www.biomedcentral.com/1471-2377/9/36/prepub

Publish with BioMed Central and every scientist can read your work free of charge

"BioMed Central will be the most significant development for disseminating the results of biomedical research in our lifetime. "

Sir Paul Nurse, Cancer Research UK

Your research papers will be:

- available free of charge to the entire biomedical community

- peer reviewed and published immediately upon acceptance

- cited in PubMed and archived on PubMed Central

- yours - you keep the copyright 